\title{
Editorial
}

\section{Some Surprising Twists on the Road to Discovering the Contribution of Rare Variants to Complex Diseases}

\author{
Duncan C. Thomas \\ University of Southern California, Los Angeles, Calif., USA
}

The advent of next-generation sequencing (NGS) offers the prospect of discovering associations with rare variants that are not effectively tagged by currently available chip technologies [1], but has posed major challenges to the statistical genetics community. Aside from the sheer logistical problems of storage, cleaning, and processing such massive data, some fundamentally new methodological issues arise, driven primarily by the extremely sparse data on any given rare variant, the weak linkage disequilibrium among them, the huge multiple comparisons penalty, and the uncertainty in genotype calls, particularly for low-coverage data (e.g. Kim et al. [2] in this issue). Rising to these challenges, the statistical genetics community has developed an incredible diversity of techniques for studying rare variants, with several new papers appearing in each of the major journals each issue over the past couple of years. (A recent PubMed search for 'rare variants' AND 'next generation sequencing' yielded 125 references, all but one since 2010, and there are doubtless more!) The current issue of Human Heredity offers an interesting sampling of some of the latest ideas. As these are primary research papers, not reviews, the reader may still feel somewhat at a loss as to how to proceed in the face of such a diversity of approaches. Fortunately, there are a few recent reviews that may be some help [1,3-7], although in such a rapidly evolving field even these excellent articles may soon be out of date. Rather than attempt yet another general review, I just wished to make a few general observations about some surprising trends in the field and offer some of my own personal opinions.

\section{Theoretical Foundations}

The first observation is that much of this literature represents something of a 'cottage industry' of trial-and-error research: investigators propose a novel test - often a minor tweak on an earlier method - and by simulation compare its performance with one or more alternatives in the hopes of finding some circumstance(s) under which the new method outperforms the competition (or at least does about as well, while being computationally more efficient). Most 'burden tests' that apply standard tests of association (e.g. logistic regression) to a covariate constructed as some kind of a weighted sum of rare variant counts fall into this class. Since we do not know the true state of nature, there is no formal basis for predicting the optimal weights, but one hopes to find a choice that performs well across a broad range of circumstances. For ex-

\section{KARGER}

E-Mail karger@karger.com

www.karger.com/hhe
(C) 2013 S. Karger AG, Basel

0001-5652/12/0744-0113\$38.00/0
Duncan C. Thomas

Division of Biostatistics, Keck School of Medicine

University of Southern California

Los Angeles, CA 90089 (USA)

E-Mail dthomas@usc.edu 
ample, a simple unweighted sum [8] might be expected to perform well if effect sizes are similar, whereas a weighted sum with weights varying inversely with minor allele frequency [9] might generally perform better if rare variants tend to have larger effect sizes, as might be expected under some population genetics models of selection (see Maher et al. [10] in this issue). But neither would be expected to perform well if deleterious and protective variants occur with similar frequencies [11]. In this case, some kind of model selection might be better, but then one must deal with the problem of model uncertainty, particularly if the same data are used for grouping variants and testing association. The paper by Tachmazidou et al. [12] in this issue illustrates this difficulty. What at face value seems like a clever idea of clustering variants hierarchically to choose weights for a burden-type test turned out to be less powerful than some other methods, while being computationally more intensive. The problem is that without any grounding in a statistical inference framework - frequentist or Bayesian - the choice of method is essentially arbitrary. Of course, permutation can always be relied upon to protect the type I error rate, but there is no logical basis for choosing between methods in terms of power, and permutation may be too computationally burdensome to be effective for genome-wide analyses.

Two examples of approaches that are based on some unifying statistical inference principles are those based on general linear mixed models and Bayesian methods. An early example of the former is the $\mathrm{C}_{a}$ test [13]. Under the hypothesis that a causal gene might harbor many variants with a range of effect sizes, the test simply looks for evidence of overdispersion, i.e. a larger variance in the casecontrol distribution conditional on the total number of variants than would be expected under a common binomial distribution if none of the variants had any effect. Although not presented as such by Neale et al. [13], Neyman and Scott's $C_{\alpha}$ test [14] is known to be a score test for the linear mixed model where effect sizes are treated as random effects and the parameter of interest is their variance rather than their mean [15]. More recently, the Sequence Kernel Association Test (SKAT) $[16,17]$ has received a great deal of attention, in part because it can be derived as a score test for the genetic variance in the linear mixed model, by essentially correlating the pairwise similarity of phenotypes with the pairwise similarity of genotypes, much in the spirit of earlier approaches to haplotype sharing [18-20]. Here the basic statistical foundations were laid out in an elegant pair of papers by Dan Schaid $[21,22]$, based on the theory of Hoeffding's U statistics [23].
Hierarchical modeling seems to provide another useful unifying framework. In a Bayesian context, the variant-specific effect sizes from the first (subject)-level model are again treated as random variables, which are in turn modeled in a second (variant)-level model, which can include various kinds of annotation information [24]. For rare variants, even Bayesian shrinkage of highly unstable estimates can still be unstable, albeit an improvement over simple maximum likelihood estimates by virtue of 'borrowing strength' from an assumption that they derive from some common distribution. For example, in an application to rare variants in $B R C A 1$ and $B R C A 2$ in the WECARE study of second breast cancers, Capanu et al. [25] showed how even variants that appeared in only a single case or control can have effects estimated by combining them with other rare variants that shared similar bioinformatic profiles. In the spirit of the burden tests, an alternative approach is to assign each variant to deleterious, protective, or null categories with weights $+1,-1$, or 0 , respectively, and then simply test association of the resulting 'burden' index with disease. Quintana et al. [26] addressed the resulting problem of model uncertainty in a Bayes model averaging framework [27], allowing inference on both the posterior probability that each variant is causal (averaging over all possible combinations of variants and their signs) and on the posterior probabilities of each model. In the current issue, they have extended this approach to include external annotation information [28], now using it in a model for the probability that a variant has a non-null effect rather than its effect size.

\section{Pathways and External Data}

The second interesting observation is the convergence of hypothesis-based and agnostic philosophies. Genomewide association studies (GWAS) were born out of a frustration with the lack of success of candidate-gene (or candidate-pathway)-based studies [29]. The general consensus was that the poor track record of replicating reported genetic associations was partly the fault of our inability to pick good candidates, with (in hindsight) most of the reported associations having very low prior credibility considering the large number of possible candidates that could have been tested. With the advent of chip and beadarray technologies and the completion of the HapMap Project finally making GWAS possible, these were widely touted as 'hypothesis-free' or 'agnostic' approaches that had the potential to discover novel genetic associations 
that might never have occurred to anyone. And indeed, the approach appears to have been wildly successful, with thousands of genome-wide significant and replicated associations having been reported for hundreds of diseases [30]. Of course, much apparent genetic variation remains to be discovered [31]. To this end, researchers are again looking to biological pathways as a way of mining the 'lower Manhattan' for combinations of variants, genes, pathways, or their interactions that might in the aggregate be significant even if individually they are not [32]. The workhorse of this class of approaches has been the gene set enrichment analysis [33], but as with burden tests, many of these approaches - both for extracting biofeatures from the wealth of -omics databases and for mining the genetic association data - are more algorithmic in nature than grounded in statistical principles.

\section{Family-Based Designs}

The third surprising trend is the resurgence of interest in family-based designs [34-39], which largely fell out of favor during the candidate gene and GWAS eras. Although family-based designs were understood to have some advantage for protection from bias due to population stratification, the techniques of genomic control were found to be quite effective at overcoming this problem, and the loss of statistical efficiency due to 'overmatching' when using related controls was felt not to be worth it. Now as we start looking for associations with rare variants, there is a growing recognition that familybased designs offer the possibility of exploiting information about the co-segregation of variants with disease as one more piece of evidence to rank variants for potential causality - information that is not available in a conventional case-control design using unrelated individuals. Part of the reason for the revival of family studies is that the spectrum of disease-causing variants that is likely to be uncovered by NGS is probably intermediate between those found by GWAS and those responsible for Mendelian disorders, and it seems likely that causal genes may harbor many different mutations, with different families segregating different variants - situations for which linkage analysis was so successful. Several contributions in this issue [40-43] illustrate this approach. The recent extension of SKAT to family data $[44,45]$ offers one way to exploit this reasoning to evaluate not just single variants but sets of variants in a gene, gene-region, or pathway.

Other advantages of family-based designs include considerably improved imputation by exploiting pre-existing

Discovering the Contribution of Rare

Variants to Complex Diseases linkage markers (essentially providing lots of 'free' sequence data on other family members) (e.g. Asimit and Zeggini [46] in this issue; but see also $[47,48]$ ) and the ability to conduct efficient 2-step analyses of the same data by exploiting between- and within-family contrasts to reduce the multiple testing burden $[49,50]$. Similar 2-step approaches have been developed for testing geneenvironment $(\mathrm{G} \times \mathrm{E})$ interactions in case-parent trios [51]. In the current issue, Kazma et al. [52] discuss other approaches to $\mathrm{G} \times \mathrm{E}$ interactions specifically aimed at rare variants, among which an extension of the SKAT approach seems to perform best (see also [53] for a similar approach). If power for testing main effects of rare variants is low to begin with, power for $\mathrm{G} \times \mathrm{E}$ effects will be even worse, but there is hope that by aggregating variants within genes or pathways, this problem can be overcome. In principle, it should be possible to combine association, sharing, and external annotation for gene- or pathwaylevel inference within some grand hierarchical modeling approach, but to date, no such approach seems to have been developed.

\section{Future Challenges}

New technologies usually spur the development of novel analytical methods. We saw this with the advent of GWAS genotyping technologies. The availability of NGS technologies and the general perception that much of the genetic heritability of complex diseases remains to be discovered has led to an even greater explosion of novel statistical methods. The cost of NGS is rapidly dropping towards the level of the early GWAS assays, but for now we are still at the point where clever study designs are going to be necessary $[3,39,54-56]$ until it becomes feasible to conduct massive studies on the scale of the current 'postGWAS' consortia, because the sample size requirements for detecting associations with rare variants will be even greater. Analytic challenges include ways of combining family-based and case-control approaches, incorporating bioinformatic predictors, dealing with measurement error in low-coverage data, amongst many others. Thus, it seems that methodological research for NGS studies will remain an active area of statistical genetics for some time until the next big thing arrives ... 'Integrative genomics' anybody?

\section{Acknowledgment}

Supported in part by NIH grants U01 HG005927, U19 CA148107, R01 ES019876, P30 CA014089 and P30 ES 07048. 


\section{References}

$>1$ Luo L, Boerwinkle E, Xiong M: Association 18 Beckmann L, Thomas DC, Fischer C, Changstudies for next-generation sequencing. Genome Res 2011;21:1099-1108.

2 Kim W, Londono D, Zhou L, Xing J, Nato AQ, Musolf A, Matise TC, Finch SJ, Gordon D: Single-variant and multi-variant trend tests for genetic association with next-generation sequencing that are robust to sequencing error. Hum Hered 2012;74:172-183.

-3 Feng T, Zhu X: Detecting rare variants. Methods Mol Biol 2012;850:453-464.

4 Gibson G: Rare and common variants: twenty arguments. Nat Rev Genet 2011;13:135-145.

$\checkmark 5$ Basu S, Pan W: Comparison of statistical tests for disease association with rare variants. Genet Epidemiol 2011;35:606-619.

6 Asimit J, Zeggini E: Rare variant association analysis methods for complex traits. Annu Rev Genet 2010;44:293-308.

$>7$ Lee S, Wu MC, Lin X: Optimal tests for rare variant effects in sequencing association studies. Biostatistics 2012;13:762-775.

$>8$ Morgenthaler S, Thilly WG: A strategy to discover genes that carry multi-allelic or monoallelic risk for common diseases: a cohort allelic sums test (CAST). Mutat Res 2007;615: 28-56.

$\checkmark 9$ Madsen BE, Browning SR: A groupwise association test for rare mutations using a weighted sum statistic. PLoS Genetics 2009; 5:e1000384

10 Maher MC, Uricchio LH, Torgerson DG, Hernandez RD: Population genetics of rare variants and complex diseases. Hum Hered 2012;74:118-128.

-11 Hoffmann TJ, Marini NJ, Witte JS: Comprehensive approach to analyzing rare genetic variants. PLoS One 2010;5:e13584.

12 Tachmazidou I, Morris A, Zeggini E: Rare variant association testing for next-generation sequencing data via hierarchical clustering. Hum Hered 2012;74:165-171.

$\checkmark 13$ Neale BM, Rivas MA, Voight BF, Altshuler D, Devlin B, Orho-Melander M, Kathiresan S, Purcell SM, Roeder K, Daly MJ: Testing for an unusual distribution of rare variants. PLoS Genetics 2011;7:e1001322.

14 Neyman J, Scott E: On the use of c(alpha) optimal tests of composite hypotheses. Bull Internat Statist Inst 1966;41:477-497.

15 Kocherlakota S, Kocherlakota K: Neyman's c(alpha) test and Rao's efficient score test for composite hypotheses. Statist Prob Lett 1991; 11:499-493.

16 Wu MC, Lee S, Cai T, Li Y, Boehnke M, Lin $\mathrm{X}$ : Rare-variant association testing for sequencing data with the sequence kernel association test. Am J Hum Genet 2011;89:82-93.

$\checkmark 17$ Lee S, Emond MJ, Bamshad MJ, Barnes KC, Rieder MJ, Nickerson DA, Christiani DC, Wurfel MM, Lin X: Optimal unified approach for rare-variant association testing with application to small-sample case-control wholeexome sequencing studies. Am J Hum Genet 2012;91:224-237. Claude J: Haplotype sharing analysis using mantel statistics. Hum Hered 2005;59:67-78.

19 Tzeng JY, Zhang D: Haplotype-based association analysis via variance-components score test. Am J Hum Genet 2007;81:927-938.

20 Allen AS, Satten GA: A novel haplotype-sharing approach for genome-wide case-control association studies implicates the calpastatin gene in Parkinson's disease. Genet Epidemiol 2009;33:657-667.

21 Schaid DJ: Genomic similarity and kernel methods ii: methods for genomic information. Hum Hered 2010;70:132-140.

22 Schaid DJ: Genomic similarity and kernel methods i: advancements by building on mathematical and statistical foundations.

23 Hoeffding: A class of statistics with asymptotically normal distributions. Ann Math Stat 1948;19:293-325.

$>24$ Lewinger JP, Conti DV, Baurley JW, Triche TJ, Thomas DC: Hierarchical Bayes prioritization of marker associations from a genomewide association scan for further investigation. Genet Epidemiol 2007;31:871-882.

25 Capanu M, Concannon P, Haile RW, Bernstein L, Malone KE, Lynch CF, Liang X, Teraoka SN, Diep AT, Thomas DC, Bernstein JL, Begg CB: Assessment of rare BRCA1 and BRCA2 variants of unknown significance using hierarchical modeling. Genet Epidemiol 2011;35:389-397.

26 Quintana MA, Berstein JL, Thomas DC, Conti DV: Incorporating model uncertainty in detecting rare variants: the Bayesian risk index. Genet Epidemiol 2011;35:638-649.

$>27$ Raftery AE, Madigan D, Hoeting JA: Bayesian model averaging for linear regression models. J Am Statist Assoc 1997;92:179-191.

28 Quintana MA, Schumacher FR, Casey G, Bernstein JL, Li L, Conti DV: Incorporating prior biologic information for high-dimensional rare variant association studies. Hum Hered 2012;74:184-195.

29 Ioannidis JP, Ntzani EE, Trikalinos TA, Contopoulos-Ioannidis DG: Replication validity of genetic association studies. Nat Genet 2001;29:306-309. mos EM, Mehta JP, Collins FS, Manolio TA: Potential etiologic and functional implications of genome-wide association loci for human diseases and traits. Proc Natl Acad Sci USA 2009; 106:9362-9367.

31 Manolio TA, Collins FS, Cox NJ, Goldstein DB, Hindorff LA, Hunter DJ, McCarthy MI, Ramos EM, Cardon LR, Chakravarti A, Cho JH, Guttmacher AE, Kong A, Kruglyak L, Mardis E, Rotimi CN, Slatkin M, Valle D, Whittemore AS, Boehnke M, Clark AG, Eichler EE, Gibson G, Haines JL, Mackay TF, McCarroll SA, Visscher PM: Finding the missing heritability of complex diseases. $\mathrm{Na}$ ture 2009;461:747-753. Hum Hered 2010;70:109-131.

30 Hindorff LA, Sethupathy P, Junkins HA, Ra-
32 Wang K, Li M, Hakonarson H: Analysing biological pathways in genome-wide association studies. Nat Rev Genet 2010;11:843-854.

33 Subramanian A, Tamayo P, Mootha VK, Mukherjee S, Ebert BL, Gillette MA, Paulovich A, Pomeroy SL, Golub TR, Lander ES Mesirov JP: Gene set enrichment analysis: a knowledge-based approach for interpreting genome-wide expression profiles. Proc Natl Acad Sci USA 2005;102:15545-15550.

-34 Bailey-Wilson JE, Wilson AF: Linkage analysis in the next-generation sequencing era. Hum Hered 2011;72:228-236.

$>35$ Wijsman EM: The role of large pedigrees in an era of high-throughput sequencing. Hum Genet 2012;131:1555-1563

36 Ionita-Laza I, Ottman R: Study designs for identification of rare disease variants in complex diseases: the utility of family-based designs. Genetics 2011;189:1061-1068.

37 Shi G, Rao DC: Optimum designs for nextgeneration sequencing to discover rare variants for common complex disease. Genet Epidemiol 2011;35:572-579.

>38 Zhu X, Feng T, Li Y, Lu Q, Elston RC: Detecting rare variants for complex traits using family and unrelated data. Genet Epidemiol 2010; 34:171-187.

39 Zhu Y, Xiong M: Family-based association studies for next-generation sequencing. Am J Hum Genet 2012;90:1028-1045.

40 Perdry H, Müller-Myhsok B, Clerget-Darpoux F: Using affected sib-pairs to uncover rare disease variants. Hum Hered 2012;74: 129-141.

$>41$ Marchani EE, Chapman NH, Cheung CYK, Ankenman K, Stanaway IB, Coon HH, Nickerson D, Bernier R, Brkanac Z, Wijsman EM: Identification of rare variants from exome sequence in a large pedigree with autism. Hum Hered 2012;74:153-164.

42 Génin E, Sahbatou M, Gazal S, Babron MC, Perdry H, Leutenegger AL: Could inbred cases identified in GWAS data succeed in detecting rare recessive variants where affected sib-pairs have failed? Hum Hered 2012;74: 142-152

43 Jouan L, Gauthier J, Dion PA, Rouleau GA: Rare variants in complex traits: novel identification strategies and the role of de novo mutations. Hum Hered 2012;74:215-225.

44 Schifano ED, Epstein MP, Bielak LF, Jhun MA, Kardia SLR, Peyser PA, Lin X: SNP set association analysis for familial data. Genet Epidemiol 2012:797-810.

45 Chen H, Meigs JB, Dupuis J: Sequence kernel association test for quantitative traits in family samples. Genet Epidemiol 2013;37:196204.

46 Asimit JL, Zeggini E: Imputation of rare variants in next-generation association studies. Hum Hered 2012;74:196-204.

47 Burdick JT, Chen WM, Abecasis GR, Cheung VG: In silico method for inferring genotypes in pedigrees. Nat Genet 2006;38:1002-1004. 
48 Li Y, Willer C, Sanna S, Abecasis G: Genotype imputation. Annu Rev Genomics Hum Genet 2009; 10:387-406

49 Van Steen K, McQueen MB, Herbert A, Raby B, Lyon H, DeMeo DL, Murphy A, Su J, Datta S, Rosenow C, Christman M, Silverman EK, Laird NM, Weiss ST, Lange C: Genomic screening and replication using the same data set in family-based association testing. Nat Genet 2005;37:683-691.

50 Wason JMS, Dudbridge F: A general framework for two-stage analysis of genome-wide association studies and its application to casecontrol studies. Am J Hum Genet 2012;90: 760-773.
51 Gauderman WJ, Thomas DC, Murcray C, Conti DV, Li D, Lewinger JP: Efficient genome-wide association testing of gene-environment interaction in case-parent trios. Am J Epidemiol 2010;172:116-122.

52 Kazma R, Cardin NJ, Witte JS: Does accounting for gene-environment interactions help uncover association between rare variants and complex diseases? Hum Hered 2012;74: 205-214.

53 Tzeng JY, Zhang D, Pongpanich M, Smith C, McCarthy MI, Sale MM, Worrall BB, Hsu FC, Thomas DC, Sullivan PF: Studying gene and gene-environment effects of uncommon and common variants on continuous traits: a marker-set approach using gene-trait similarity regression. Am J Hum Genet 2011;89:277288.
54 Thomas DC, Casey G, Conti DV, Haile RW, Lewinger JP, Stram DO: Methodological issues in multistage genome-wide association studies. Stat Sci 2009;24:414-429.

55 Cirulli ET, Goldstein DB: Uncovering the roles of rare variants in common disease through whole-genome sequencing. Nat Rev Genet 2010;11:415-425.

56 Mechanic LE, Chen HS, Amos CI, Chatterjee N, Cox NJ, Divi RL, Fan R, Harris EL, Jacobs K, Kraft P, Leal SM, McAllister K, Moore JH, Paltoo DN, Province MA, Ramos EM, Ritchie MD, Roeder K, Schaid DJ, Stephens M, Thomas DC, Weinberg CR, Witte JS, Zhang S, Zollner S, Feuer EJ, Gillanders EM: Next generation analytic tools for large scale genetic epidemiology studies of complex diseases. Genet Epidemiol 2012;36:22-35. 